\title{
COVID-19-associated acute disseminated encephalomyelitis (ADEM)
}

\author{
Timothy Parsons $s^{1,2}$ - Sarah Banks ${ }^{1,2} \cdot$ Chay Bae ${ }^{2} \cdot$ Joel Gelber $^{1} \cdot$ Hussein Alahmadi ${ }^{1} \cdot$ Matthew Tichauer $^{1,2}$
}

Received: 6 May 2020 / Revised: 21 May 2020 / Accepted: 25 May 2020 / Published online: 30 May 2020

(c) Springer-Verlag GmbH Germany, part of Springer Nature 2020

\begin{abstract}
A 51-year-old woman with COVID-19 infection developed coma and an impaired oculocephalic response to one side. MRI of the brain demonstrated acute multifocal demyelinating lesions, and CSF testing did not identify a direct cerebral infection. High-dose steroids followed by a course of IVIG was administered, and the patient regained consciousness over the course of several weeks. As more patients reach the weeks after initial infection with COVID-19, acute disseminated encephalomyelitis should be considered a potentially treatable cause of profound encephalopathy or multifocal neurological deficits.
\end{abstract}

Keywords ADEM $\cdot$ Post-infectious $\cdot$ COVID-19 $\cdot$ Coronavirus $\cdot$ Demyelinating disease

\section{Dear Editor,}

Neurological symptoms with SARS-CoV-2 infection (COVID-19) have been commonly encountered, but specific syndromes are only beginning to be described. Here, we report a patient with coma, an impaired unilateral oculocephalic response, and left hemiparesis. MRI, CSF, and her clinical course were consistent with acute disseminated encephalomyelitis (ADEM).

A 51-year-old woman presented to a local hospital with dyspnea, fever, and vomiting. She had no pertinent neurological history. She was febrile, tachycardic, and hypoxic. Chest X-ray revealed extensive patchy airspace opacification. SARS-CoV-2 PCR from a nasopharyngeal swab was positive. She was intubated, and was maintained on sedative drips. On hospital day 18 , she was transferred to our facility due to persistent fever and failure to progress clinically.

Sedatives were held on arrival. Neurological exam was notable for unresponsiveness (GCS 3). Pupils were equal and reactive to light, corneal responses were intact, and the oculocephalic response to the left was impaired. Muscle tone was flaccid throughout, and the extremities did not move

Timothy Parsons

timothy.parsons@hhchealth.org

1 The Hospital of Central Connecticut, Hartford Healthcare, 100 Grand St., New Britain, CT 06052, USA

2 University of Connecticut School of Medicine, Farmington, CT, USA spontaneously or to noxious stimuli. Deep tendon reflexes were depressed, and plantar responses were mute.

An MRI of the brain done with and without gadolinium contrast on hospital day 24 showed scattered hyperintense lesions on FLAIR imaging in deep hemispheric and juxtacortical white matter. These lesions were hyperintense on diffusion weighted imaging (DWI), and a minority showed subtle restricted diffusion on the apparent diffusion coefficient (ADC), indicating acuity, but not consistent with infarction (Figs. 1, 2). A FLAIR hyperintensity in the left frontal juxtacortical white matter showed mild enhancement with gadolinium contrast (Fig. 3). There was a small amount of intraventricular hemorrhage (IVH) layering in the occipital horns of both lateral ventricles. The Gradient Echo sequence did not show evidence of parenchymal hemorrhage.

Lumbar puncture was atraumatic. CSF analysis demonstrated 1 white blood cell, 2095 red blood cells with xanthochromia, $62 \mathrm{mg} / \mathrm{dl}$ protein, $56 \mathrm{mg} / \mathrm{dl}$ glucose. Bacterial culture, fungal culture, and a PCR panel (including HSV, VZV, EBV, and CMV) were negative. There were four oligoclonal bands, present in both serum and CSF. SARS-CoV-2 was not detected by qualitative PCR.

Renal function was normal throughout the hospital course. Transaminases were mildly elevated. CT angiogram of the Head and Neck was normal, excluding ruptured aneurysm as a cause of the IVH. Transthoracic echocardiogram and serum tests for ANA, ANCA, Syphilis, HIV, and Aquaporin 4 antibody were negative or normal. EEG demonstrated diffuse slowing. 

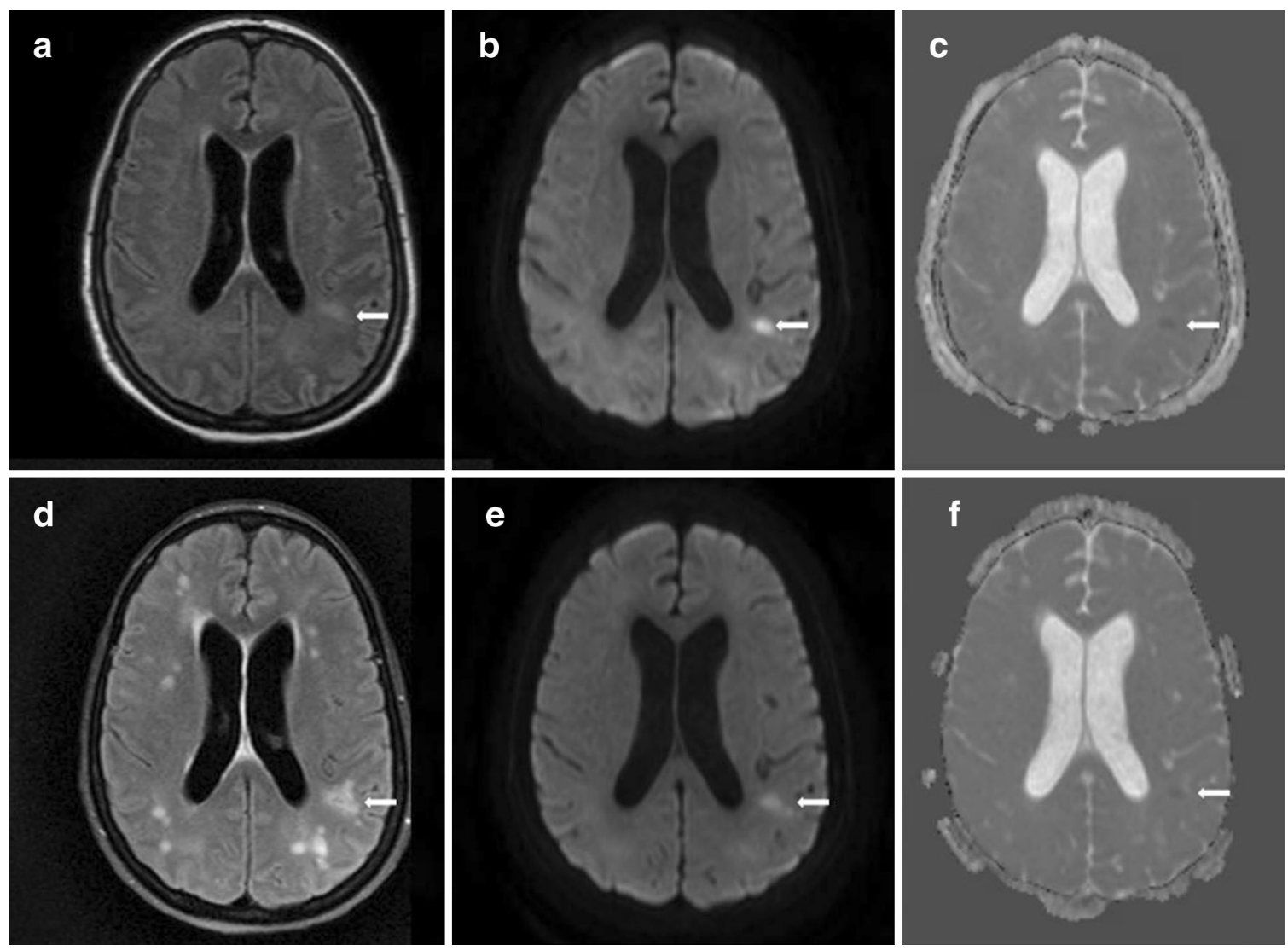

Fig. 1 MRI brain on hospital day 29 showed FLAIR hyperintensities in the deep hemispheric, periventricular, and juxtacortical white matter (arrow, a), mostly hyperintense on diffusion weighted imaging (DWI) (arrow, b), and some show subtle restricted diffusion on apparent diffusion coefficient (ADC) imaging (arrow, c). Repeat MRI

Methylprednisolone $1 \mathrm{~g}$ IV daily for 5 days was administered for presumed ADEM. Exams with sedation held were grossly unchanged. Intravenous Immunoglobulin (IVIG) $0.4 \mathrm{~g} / \mathrm{kg}$ daily was administered for 5 days starting on hospital day 31 . Alertness improved gradually and on hospital day 36 she started to follow simple commands. Left hemiparesis became evident. On hospital day 39 , she was extubated and was able to begin speaking, and on hospital day 59, she was fully oriented. MRI of the brain with and without gadolinium contrast was repeated on hospital days 29,38 , and 58 with an increase in the number and distribution of FLAIR lesions (Figs. 1, 2) and a new right frontal enhancing lesion (Fig. 3). brain on hospital day 58 showed an increased number and distribution of FLAIR hyperintensities in the hemispheric white matter (arrow, d), with persistence of some previous lesions on DWI (arrow, e) and $\mathrm{ADC}$ (arrow, $\mathbf{f}$ ), but no new lesions on these sequences

ADEM is a rare demyelinating disease, often post-viral, and more commonly seen in children than adults. Clinically, it is heterogeneous, generally causing encephalopathy and multifocal deficits. MRI typically demonstrates FLAIR hyperintensities in deep white matter and at the grey/white matter interface. Post-contrast enhancement is not always present but is often punctate or rim enhancing. Diffusion restriction can be seen, especially early in the course of the disease [1, 2]. ADEM lesions can be hemorrhagic [3, 4], but the etiology of the intraventricular hemorrhage in this case is unclear. Anticoagulation had not been utilized, blood pressure had been well controlled, there was no laboratory evidence of coagulopathy, and she had not suffered trauma. 

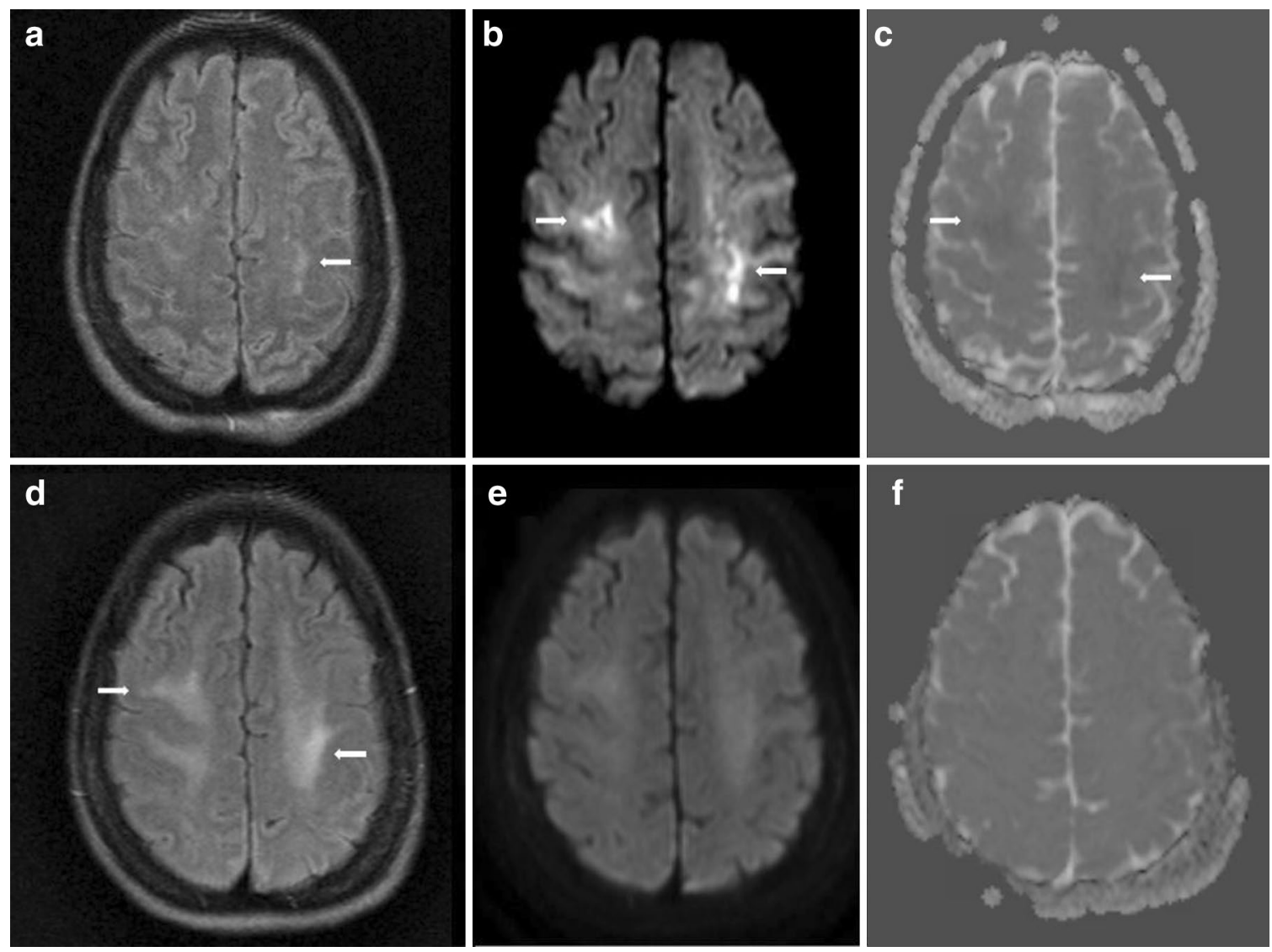

Fig. 2 MRI brain on hospital day 24 showed small FLAIR hyperintensities in the juxtacortical white matter (arrow, a), more widespread hyperintensities on diffusion weighted imaging (DWI) (arrows, b), with subtle restricted diffusion on apparent diffusion coefficient

The location and evolution of lesions on MRI, sparse contrast enhancement, clinical exam, and CSF are all consistent with an acute demyelinating event, and the clinical situation in this case, weeks after an acute viral infection, is favorable for the development of ADEM. ADEM has
(ADC) imaging (arrows, c). Repeat MRI brain on hospital day 58 showed poorly defined FLAIR hyperintensities in the juxtacortical white matter (arrow, d), with resolution of the signal abnormalities on DWI (e) and ADC (f) sequences

been reported in a child following a coronavirus infection [5]. As more patients reach the weeks after initial infection with COVID-19, ADEM should be considered a potentially treatable cause of profound encephalopathy or multifocal neurological deficits. 

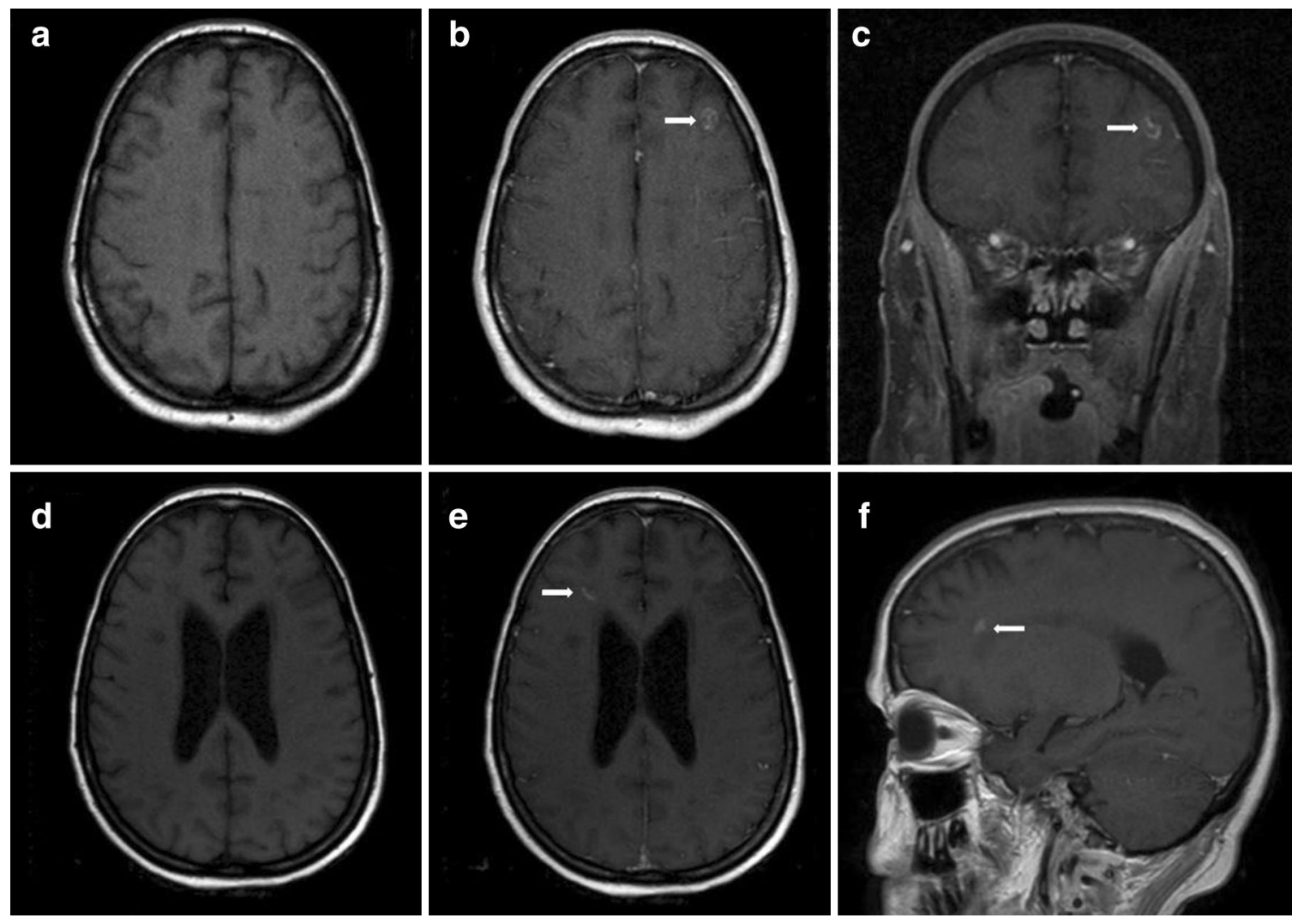

Fig. 3 MRI brain on hospital day 24 showed a small contrast enhancing lesion (arrows) in the left frontal lobe at the grey-white interface (a T1 axial. b T1 axial post-gadolinium. c T1 coronal post-gadolin-

Author contributions TP MD, SB MD, CB DO, JG MD, HA MD, MT MD: drafted and revised the manuscript for intellectual content.

Funding Not applicable.

Availability of data and material Not applicable.

\section{Compliance with ethical standards}

Conflicts of interest On behalf of all authors, the corresponding author states that there is no conflict of interest.

\section{Ethics approval Not applicable.}

\section{Consent to participate Not applicable.}

Consent for publication Consent to publish acquired from legal next of kin and the patient.

Code availability Not applicable. ium). MRI brain on hospital day 58 showed a small contrast enhancing lesion (arrows) in the right frontal white matter (d T1 axial, e T1 axial post-gadolinium, $\mathbf{f} \mathrm{T} 1$ sagittal post-gadolinium.)

\section{References}

1. Marin SE, Callen DJ (2013) The magnetic resonance imaging appearance of monophasic acute disseminated encephalomyelitis: an update post application of the 2007 consensus criteria. Neuroimaging Clin N Am 23:245-266

2. Balasubramanya KS, Kovoor JM, Jayakumar PN et al (2007) Diffusion-weighted imaging and proton MR spectroscopy in the characterization of acute disseminated encephalomyelitis. Neuroradiology 49:177-183

3. Tenembaum S, Chamoles N, Fejerman N (2002) Acute disseminated encephalomyelitis: a long-term followup study of 84 pediatric patients. Neurology 59:1224-1231

4. Kuperan S, Ostrow P, Landi MK, Bakshi R (2003) Acute hemorrhagic leukoencephalitis vs ADEM: FLAIR MRI and neuropathology findings. Neurology 60:721

5. Yeh EA, Collins A, Cohen M, Duffner PK, Faden H (2004) Detection of coronavirus in the central nervous system of a child with acute disseminated encephalomyelitis. Pediatrics 113:e73-76 